Europhys. Lett., 50 (1), pp. 54-60 (2000)

\title{
Phase diagram of a classical fluid in a quenched random potential
}

\author{
F. Thalmann ${ }^{1,2}$, C. Dasgupta ${ }^{1}$ and D. Feinberg ${ }^{2}$ \\ 1 Department of Physics, Indian Institute of Science - Bangalore 560012, India and \\ Condensed Matter Theory Unit, Jawaharlal Nehru Centre for Advanced Scientific \\ Research - Bangalore 560064, India \\ 2 Laboratoire d'Etudes des Propriétés Electroniques des Solides(*) \\ Centre National de la Recherche Scientifique - BP166, 38042 Grenoble Cedex 9, France
}

(received 6 August 1999; accepted in final form 17 January 2000)

PACS. 64.70.Pf - Glass transitions.

PACS. 64.70.Dv - Solid-liquid transitions.

PACS. 05.20.Jj - Statistical mechanics of classical fluids.

\begin{abstract}
We consider the phase diagram of a classical fluid in the presence of a random pinning potential of arbitrary strength. Introducing replicas for averaging over the quenched disorder, we use the hypernetted chain approximation to calculate the correlations in the replicated liquid. The freezing transition of the liquid into a nearly crystalline state is studied using a density functional approach, and the liquid-to-glass transition is studied using a phenomenological replica symmetry breaking approach introduced by Mézard and Parisi. The first-order liquid-to-crystal transition is found to change to a continuous liquid-to-glass transition as the strength of the disorder is increased above a threshold value.
\end{abstract}

The equilibrium phase diagram of a classical system of interacting particles in the presence of quenched pinning potential is a subject of much current interest, in view of understanding the behavior of various systems such as magnetic bubble arrays [1], Wigner crystals of electrons [2], and flux lines in the mixed phase of high- $T_{\mathrm{c}}$ superconductors [3]. In particular, for layered type-II superconductors such as $\mathrm{Bi}_{2} \mathrm{Sr}_{2} \mathrm{CaCu}_{2} \mathrm{O}_{8}$ in a magnetic field $H$ perpendicular to the layers, the $(T, H)$ phase diagram is especially interesting. The flux lines in these materials may be viewed as columns of interacting "pancake" vortices residing on the layers, and the properties of the mixed phase may be described in terms of the classical statistical mechanics of these point-like objects. At low enough fields, a first-order melting transition separates an ordered vortex lattice from a disordered "vortex liquid" state [4]. Existing theoretical studies $[5,6]$ suggest that weak point disorder only slightly distorts the crystalline state, leading to the observed "Bragg glass" phase with quasi-long-range translational order [7]. The freezing line of a layered system of pancake vortices in the presence of weak point pinning has been

$\left(^{*}\right)$ Laboratoire associé à l'Université Joseph Fourier.

(C) EDP Sciences 
studied theoretically [8], assuming the solid phase to be a harmonic crystal. When the magnetic field is increased, or equivalently with increasing disorder [9], the experimental transition becomes continuous $[4,10]$, and the "Bragg glass" is replaced by an amorphous state, which is believed to be a vortex glass $[11,12]$.

This scenario could be a very general one, e.g. the first-order liquid-to-crystal transition in a three-dimensional ( $3 d$ ) fluid may be driven by quenched disorder into a continuous liquidto-glass transition. In the present letter, we consider, rather than vortices, a simpler and better documented system, namely a fluid of hard spheres in a random pinning potential of arbitrary strength. This is also a richer system since it offers the possibility of an intrinsic, though metastable, glassy phase, and the stabilization of this phase by quenched disorder is of considerable interest. We expect that our results could be generalized and applied to the systems listed above. Using two "mean-field"-type approaches based on the "replicated liquid formalism" $[8,13,14]$, we obtain a phase diagram in the density $(\rho)$-disorder $(\delta)$ plane which shows crystalline, liquid and glassy phases. One expects that: i) the transition between crystal and liquid phases remains first order, while the transition point itself is shifted with increasing disorder; ii) from earlier work [8] and arguments [15] based on the Lindemann criterion, the liquid phase is favored by the disorder. Our phase diagram is consistent with these expectations. We also find that the first-order crystallization transition is replaced by a continuous glass transition as the disorder strength is increased above a threshold value.

We consider a fluid of monodisperse hard spheres in the presence of an external, shortrange, random pinning potential $\Phi(\vec{r})$. In the absence of disorder, the crystal becomes the stable phase at $\eta=\eta_{f} \simeq 0.49(\rho \simeq 0.94)$ where the packing fraction $\eta$ and the dimensionless density $\rho$ are related by $\eta=\pi \rho / 6$ (spheres of diameter 1 ). If the hard-sphere fluid is kept in a "supercompressed" state, then slow dynamics effects, analogous to those occurring near the structural glass transition in supercooled liquids, are observed [16]. Whether a true thermodynamic glass transition occurs in this system at a packing fraction lower than that for random close packing $\left(\eta_{\mathrm{rcp}} \simeq 0.64\right)$ is unclear [17]. In analogy with experiments, one may define the "glass transition" to occur at the point where the characteristic relaxation time in the fluid exceeds a given value $\tau_{\mathrm{c}}$. Then, the phase diagram of the pure hard-sphere fluid would show a crystalline phase that is stable for $\eta>0.49$, and at $\eta=\eta_{\mathrm{g}}$, just below $\eta_{\mathrm{rcp}}$, a transition from the (metastable) liquid to a (metastable) glassy phase.

The random potential $\Phi(\vec{r})$ is assumed to be Gaussian with an exponentially decreasing correlator $\mathcal{V}\left(\vec{r}-\vec{r}^{\prime}\right)=\overline{\Phi(\vec{r}) \Phi\left(\vec{r}^{\prime}\right)}=\Delta \cdot \exp \left[-\left(\left|\vec{r}-\vec{r}^{\prime}\right|^{2}\right) / \xi^{2}\right] ; \bar{\Phi}=0(-$ means averaging over the probability distribution of $\Phi)$, and a correlation length $\xi=0.25$. This choice implies that local minima of $\Phi$ contain at most one sphere. The parameter $\Delta$ measures the strength of the disorder. As soon as an external potential is introduced, the temperature becomes a relevant parameter: the disorder may be considered perturbative if $\delta \equiv \beta^{2} \Delta \ll 1 ; \beta=1 / k_{\mathrm{B}} T$, while $\delta \gg 1$ corresponds to the strong pinning limit.

We make use of the "replicated liquid formalism", introduced for studying fluids in porous media [13], flux-lattice melting [8], and more recently, the structural glass transition [14]. The replica method is used for averaging over the disorder and mapping the problem of an inhomogeneous liquid in a random potential onto the problem of a homogeneous, multicomponent mixture on which standard tools of liquid theory can be applied. Before averaging over the disorder, the particles are coupled to $\Phi(\vec{r})$ and interact via a hard-sphere repulsion. After introducing $n$ copies ("replicas", labelled $a, b=1, \ldots, n$ ) of the system and averaging over the Gaussian disorder, one is left with a mixture of $n$ components, interacting with $v_{*}(r)=-\beta \mathcal{V}(r)$ plus a hard-sphere repulsion if the two particles belong to the same replica, and with $v_{0}(r)=-\beta \mathcal{V}(r)$ otherwise.

The structure of the replicated liquid is expressed in terms of the pair correlation functions 
$g_{a b}(r)=1+h_{a b}(r)$ between particles of replicas $a$ and $b$. Direct correlations functions $c_{a b}(r)$ are introduced, related to the $h_{a b}$ via the Ornstein-Zernike relation, expressed in Fourier space as

$$
\forall a, b ; \tilde{h}_{a b}(q)=\tilde{c}_{a b}(q)+\sum_{d} \tilde{h}_{a d}(q) \cdot \rho_{d} \cdot \tilde{c}_{d b}(q) .
$$

Following refs. $[8,14]$, we use the closure scheme known as the hypernetted chain (HNC) approximation in which the $n \rightarrow 0$ limit can be taken analytically. In our case, the HNC equations read

$$
g_{a b}(r)=\left(1-\delta_{a b}+\delta_{a b} \cdot \Theta(r-1)\right) \cdot \exp \left[\beta^{2} \mathcal{V}(r)+h_{a b}(r)-c_{a b}(r)\right] .
$$

At relatively low values of the packing fraction, replica symmetry is a natural assumption. Then, the set of functions $g_{a b}\left(h_{a b}\right.$ and $\left.c_{a b}\right)$ reduces to $g_{*}\left(h_{*}\right.$ and $\left.c_{*}\right)$ if $a=b$ and $g_{0}\left(h_{0}\right.$ and $\left.c_{0}\right)$ if $a \neq b$, while all the densities $\rho_{a}$ are equal to $\rho$. The function $g_{*}(r)$ looks very similar to the usual pair correlation function of the pure system. In the absence of disorder, $g_{0}(r)=1$, and it starts showing structure when $\delta$ is increased. It exhibits peaks at the maxima of $g_{*}(r)$, plus an extra peak at the origin induced by the attractive coupling between replicas.

The freezing into the nearly crystalline Bragg glass phase occurs in the replica symmetric (RS) regime. According to the standard approach of density functional theory $[8,18]$, the crystal manifests itself through a (almost) periodic modulation of the density $\rho_{\mathrm{cr}}(\vec{r})$, obeying the approximate self-consistency equation

$$
\rho_{\mathrm{cr}}(\vec{r})=\rho \cdot \exp \left[\int \mathrm{d} \vec{r} C_{e}\left(\vec{r}-\vec{r}^{\prime}\right) \cdot\left(\rho_{\mathrm{cr}}(\vec{r})-\rho\right)\right],
$$

where the function $C_{e}(\vec{r})$ stands for $\sum_{b} c_{a b}(\vec{r})=c_{*}+(n-1) \cdot c_{0}(\vec{r})=c_{*}(\vec{r})-c_{0}(\vec{r})$ for $n \rightarrow 0$ in the RS regime. The determination of the freezing line in the presence of disorder then reduces to a standard density functional calculation with a modified direct correlation function.

The glass transition in this system is studied using the phenomenological approach of Mézard and Parisi (MP) [14] who found the occurrence of one-step replica symmetry breaking (RSB) in eqs. (1), (2) in the absence of external disorder and interpreted it as the emergence of a glassy phase. MP consider a functional $\mathcal{F}$ of the pair correlation functions $g_{a b}(r)$ which yields the set of equations (1), (2) upon functional differentiation with respect to $g_{a b}$. The one-step RSB solution consists, as usual, in forming $n / m$ groups, each containing $m$ replicas [19]. Pair correlations $g_{a b}$ are set to be $g_{*}$ for $a=b, g_{1}$ for $a \neq b$ with $a, b$ in the same group, and $g_{0}$ if $a$ and $b$ are in different groups. Then, one looks for solutions making the free energy $\mathcal{F}$ stationary with respect to $g_{*}, g_{1}, g_{0}$ and $m$, with $0<m<1$ and $n=0$. By analogy with one-step RSB in mean-field spin-glass models with multispin interactions, a "dynamical transition" density $\rho_{\text {dyn }}$ is defined as the minimal density for which a RSB solution with $m=1$, stationary with respect to $g_{*}, g_{1}, g_{0}$ but not with respect to $m$, exists. The occurrence of a stationary solution with $m=1$ at a higher value of $\rho$ signals a thermodynamic glass transition.

The approach of MP relies strongly on the assumption that in the vicinity of the glass transition, the configuration space is split into an exponentially large number of "metastable states" [19]. These metastable states come into existence at the "dynamical transition" density $\rho_{\text {dyn }}$, and the thermodynamic glass transition is expected to occur at the density where the configurational entropy associated with the metastable states vanishes [20,21]. Since the concept of metastable states is itself a mean-field one borrowed from the study of infiniterange spin-glass models, it is not clear whether this description survives in real $3 d$ fluids. Nevertheless, we believe that both thermodynamics and dynamics of realistic $3 d$ fluids near the glass transition are dominated by long-lived configurations [22,23], which would be the 

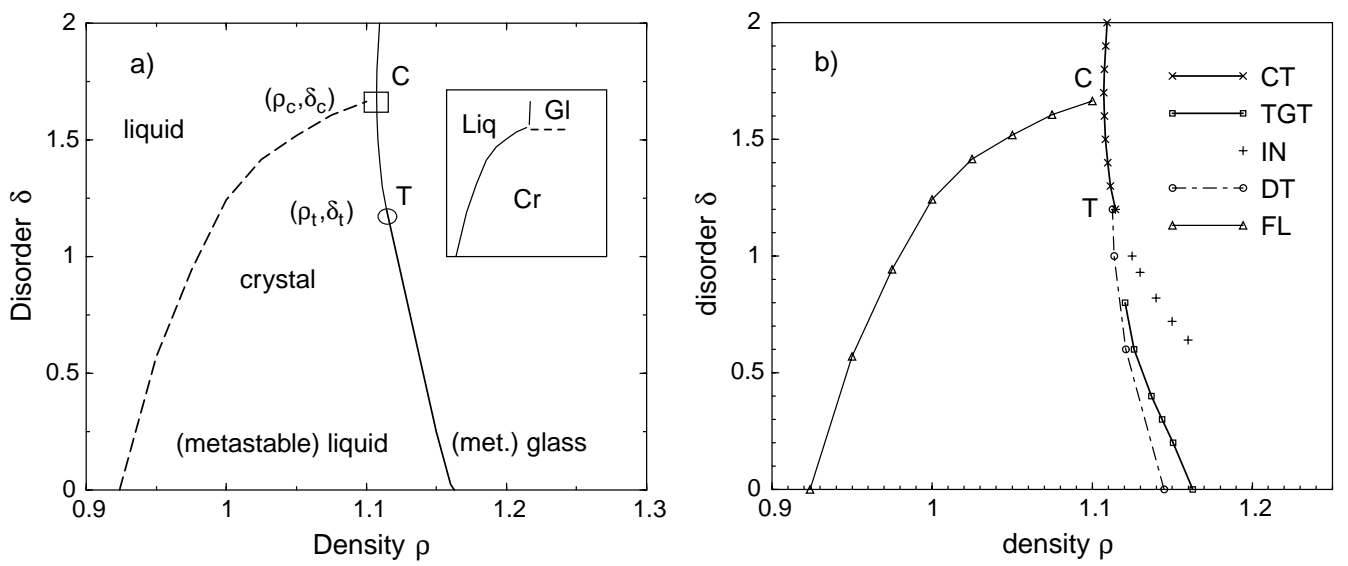

Fig. 1 - (a) The phase diagram in the density $\rho$-disorder $\delta$ plane. $\mathrm{T}$ is a tricritical point where the nature of the glass transition changes from first order to continuous, and $\mathrm{C}$ is a critical endpoint where the continuous glass transition line meets the first-order freezing line. The inset depicts the phase diagram in which only the thermodynamically stable phases are shown. (b) Various transition lines (see text for details) in the $\rho-\delta$ plane. TGT: first-order glass transition for weak disorder; CT: continuous glass transition for strong disorder; DT: dynamical transition; IN: instability of the RS solution; FL: freezing line obtained from density functional theory.

$3 d$ analog of the metastable states. Then, the method proposed by MP may be regarded as a phenomenological way of finding when these long-lived states start playing a significant role. We also note that results very similar to those of MP have been obtained in a calculation [24] that uses a different method for locating the glass transition in the pure hard-sphere system.

Due to the finite-dimensional character of the system, the "overlaps" $g_{1}(r), g_{0}(r)$ have a spatial structure which accounts for the short-range ordering of the particles in the metastable states. Roughly speaking, $g_{1}(r)$ describes short-range correlations of the average local density in a typical metastable state, and $g_{0}(r)$ represents such correlations between different metastable states $\left(g_{0}(r)=1\right.$ in the absence of disorder). We define the scalar $\mathcal{Q}=$ $\left[\int \mathrm{d} r 4 \pi r^{2}\left(g_{1}(r)-g_{0}(r)\right)^{2}\right]^{1 / 2}$ as a global order parameter. At zero disorder, the RSB transition occurs at $\rho_{\mathrm{g}} \simeq 1.16$ and is discontinuous, with a jump in the value of $\mathcal{Q}$ at the transition [25].

We have solved the replicated liquid-state equations using the method proposed by Zerah [26], and a grid of 512 points. Figure 1(a) presents our phase diagram. The thermodynamic glass transition found in [14] extends (thick line) from $\rho \simeq 1.16, \delta=0$ to a "tricritical" point $\mathrm{T}: \rho_{\mathrm{t}} \simeq 1.11, \delta_{\mathrm{t}} \simeq 1.2$. For $\delta<\delta_{\mathrm{t}}, \mathcal{Q}$ jumps discontinuously at the transition, while for $\delta>\delta_{\mathrm{t}}$ (thin line), $\mathcal{Q}$ grows continuously from zero as $\rho$ is increased across the transition line. The dashed line represents the freezing line obtained from the density functional calculation. The liquid phase is favored by the disorder and the freezing line crosses the glass transition line at $\mathrm{C}: \rho_{\mathrm{c}} \simeq 1.10, \delta_{\mathrm{c}} \simeq 1.67$. The glass phase is also favored by the disorder until $\delta \simeq 1.7$ where the liquid shows a re-entrance. The upper part of the freezing line lies in a region where the disorder is not weak $(\delta>1)$ and so the stability of the Bragg glass phase is not ensured. However, if we assume that the ordered phase remains stable in this region, then extrapolation of the freezing line into the glassy domain leads to the inset of fig. 1(a), where only stable phases are shown. As the density is increased at constant $\delta$, the system undergoes a first-order transition to a nearly crystalline state if $\delta<\delta_{\mathrm{c}}$, and a continuous glass transition for $\delta>\delta_{\mathrm{c}}$. Thus, the phase diagram exhibits a multicritical point where a 

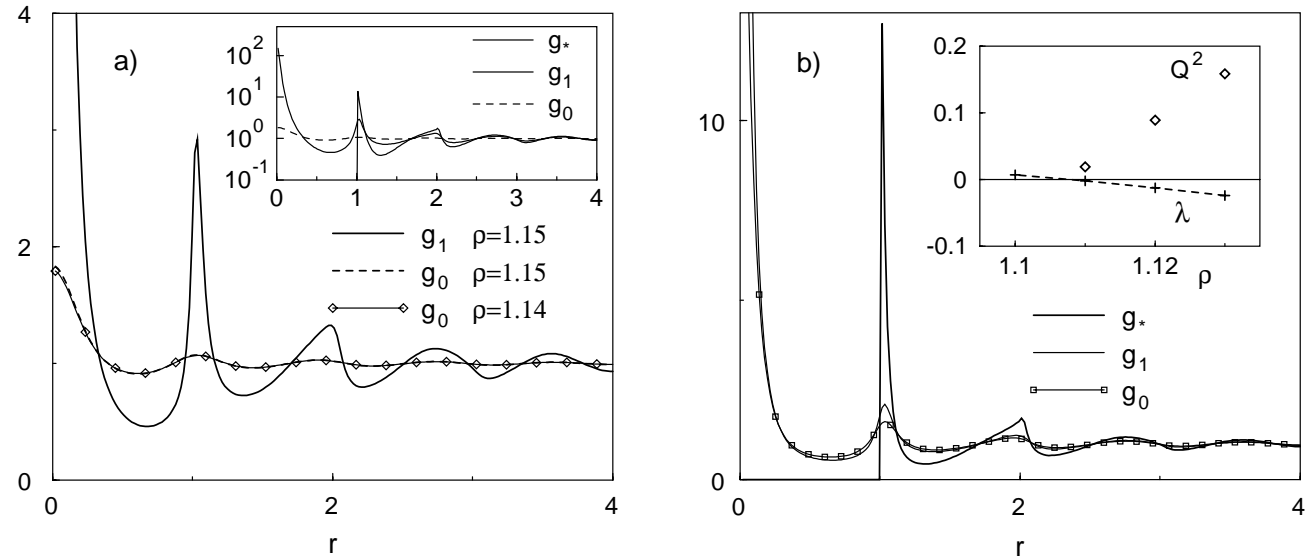

Fig. 2 - (a) HNC pair correlation functions near TGT, at $\delta=0.3$. Thick and dashed lines stand, respectively, for $g_{1}$ and $g_{0}$ at $\rho=1.15$, slightly on the glass side, while the "diamond curve" is the replica symmetric solution at $\rho=1.14$, on the liquid side. Note the jump of $\mathcal{Q}$ at the transition. Inset: $g_{*}, g_{1}, g_{0}$ at $\delta=0.3, \rho=1.15$. (b) Pair correlation functions near the continuous transition CT, at $\delta=1.8$. Inset: the "order parameter" $\mathcal{Q}$ (see text) which vanishes at the transition point. Also shown is $\lambda$, the lowest eigenvalue of the quadratic form enclosed in braces in eq. (5), which changes its sign at the same point.

line of continuous liquid-glass transition ends at a line of first-order transitions representing a liquid-crystal transition for $\delta<\delta_{\mathrm{c}}$ and a glass-crystal transition for $\delta>\delta_{\mathrm{c}}$.

We now provide some of the technical details of our calculations. The HNC free-energy per unit volume $V$ reads

$$
\begin{aligned}
\frac{2 \beta \mathcal{F}}{n V}= & \rho^{2} \int \mathrm{d} \vec{r}\left\{g_{*}\left(\ln g_{*}-1+\beta v_{*}\right)+(m-1) g_{1}\left(\ln g_{1}-1+\beta v_{0}\right)-m g_{0}\left(\ln g_{0}-1+\beta v_{0}\right)\right\}+ \\
& +\int \frac{\mathrm{d} \vec{q}}{(2 \pi)^{3}}\left\{\rho \tilde{h}_{*}-\frac{\rho^{2}}{2}\left(\tilde{h}_{*}^{2}+(m-1) \tilde{h}_{1}^{2}-m \tilde{h}_{0}^{2}\right)+\frac{1-m}{m} \ln \left(1+\rho \tilde{h}_{*}-\rho \tilde{h}_{1}\right)-\right. \\
& \left.-\frac{1}{m} \ln \left(1+\rho \tilde{h}_{*}+(m-1) \rho \tilde{h}_{1}-m \rho \tilde{h}_{0}\right)-\frac{\rho \tilde{h}_{0}}{\left[1+\rho \tilde{h}_{*}+(m-1) \rho \tilde{h}_{1}-m \rho \tilde{h}_{0}\right]}\right\}
\end{aligned}
$$

In the $n \rightarrow 0$ limit, a physically stable solution must be a minimum with respect to variations of $g_{*}$, but a maximum with respect to $g_{1}, g_{0}$. As $m \rightarrow 1$, the equations for $g_{1}$ decouple from those of $g_{*}$ and $g_{0}$, and one can get simultaneously the RSB solution $\left(g_{*}, g_{1} \neq g_{0}\right)$ and the RS one $\left(g_{*}, g_{1}=g_{0}\right)$. Both stable RS and RSB solutions exist in the domain bounded by the lines (IN, DT, $\delta=0$ ) in fig. 1(b). The thermodynamic glass transition (line TGT) occurs when $\partial \mathcal{F} /\left.\partial m\right|_{m=1}=0$, i.e. when the second term $\Phi^{\prime}\left[g_{*}, g_{1}, g_{0}\right]$ of the expansion $\mathcal{F}=\Phi\left[g_{*}, g_{0}\right]+(m-1) \cdot \Phi^{\prime}+\mathcal{O}\left((m-1)^{2}\right) \ldots$ vanishes (this is our practical criterion for determining the location of the line TGT).

Examples of RS and RSB solutions on two sides of the line TGT at $\delta=0.3$ are shown in fig. 2(a). These plots illustrate the discontinuity in $\mathcal{Q}$ across the line TGT. This discontinuity decreases with increasing $\delta$, and the transition eventually becomes continuous for $\delta \geq \delta_{\mathrm{t}}$ on the line CT (continuous transition). Figure 2(b) presents the RSB solution next to the line CT at $\delta=1.8$. The functions $g_{1}$ and $g_{0}$ look very similar, indicating that $\mathcal{Q}$ vanishes at the transition, as shown in the inset. The transition at $\delta>\delta_{\mathrm{t}}$ occurs via a bifurcation mechanism. This 
can be understood by expanding $\mathcal{F}$ to second order in $\Delta g_{*}, \Delta g_{1}, \Delta g_{0}$ around the RS solution. Defining $\Delta k_{1}=\sqrt{m(1-m)}\left(\Delta h_{1}-\Delta h_{0}\right), \Delta k_{0}=(1-m) \Delta h_{1}+m \Delta h_{0}$, and $A=1+\rho \tilde{h}_{*}-\rho \tilde{h}_{0}$, we get

$$
\frac{\beta \Delta \mathcal{F}}{n V}=\mathcal{J}\left(\Delta h_{*}, \Delta k_{0}\right)-\rho^{2} / 4\left\{\int \mathrm{d} \vec{r}\left(g_{0}^{-1}-1\right) \Delta k_{1}^{2}+\int \mathrm{d} \vec{q} /(2 \pi)^{3} A^{-2} \Delta \tilde{k}_{1}^{2}\right\},
$$

where $\mathcal{J}$ is an $m$-independent quadratic form. We have diagonalized a discrete version of the quadratic form enclosed in the braces, and found that its lowest eigenvalue $\lambda_{\text {min }}$ changes its sign, from positive (in the liquid phase) to negative (in the glass phase), just when $\mathcal{Q}$ vanishes (inset of fig. 2(b)). The RS solution turns unstable as $\lambda_{\min }$ becomes negative, providing a precise determination of the line CT. This line admits a continuation for $\delta<\delta_{\mathrm{t}}$, labelled IN (instability line) in fig. 1(b), which does not seem to have any physical meaning. A similar computation around the RSB solution enables us to determine precisely the bifurcation point at which the RSB solution, $m=1$, appears. This calculation yields the "dynamical transition" line DT. As the transition becomes continuous near $\delta \sim \delta_{\mathrm{t}}$, finding numerically the line TGT becomes very difficult because the $\Phi^{\prime}$ variations become vanishingly small. However, the fact that the lines CT, DT and IN converge to the same point $\mathrm{T}$ leads us to the conclusion that the TGT line also ends at T, as depicted in fig. 1(a).

The pure hard-sphere fluid freezes into a fcc lattice. We use the Ramakrishnan-Yussouff method [18] for expressing the density $\rho_{\mathrm{cr}}(\vec{r})$ in terms of three "order parameters" - the Fourier components, $\mu_{1,1,1}, \mu_{3,1,1}$, of the density for two sets $((1,1,1)$ and $(3,1,1))$ of reciprocal lattice vectors, and $\eta$, the fractional change in the average density:

$$
\ln \frac{\rho_{\mathrm{cr}}}{\rho}=\left[\eta \rho \tilde{C}_{e}(0)+\mu_{1,1,1} \sum_{(1,1,1)} \tilde{C}_{e}\left(\left|K_{1,1,1}\right|\right) e^{i \vec{K}_{1,1,1} \cdot \vec{r}}+\mu_{3,1,1} \sum_{(3,1,1)} \tilde{C}_{e}\left(\left|K_{3,1,1}\right|\right) e^{i \vec{K}_{3,1,1} \cdot \vec{r}}\right]
$$

We believe that this parametrization is justified in the presence of weak disorder because the nearly crystalline Bragg glass phase exhibits well-defined peaks in its structure factor. The $\mathrm{HNC}$ direct correlation function is used as input, and the coexistence line is obtained from the usual thermodynamic criterion [18]

$$
\int \mathrm{d} \vec{r}\left(\rho_{\mathrm{cr}} \cdot \ln \left(\frac{\rho_{\mathrm{cr}}}{\rho}\right)-\rho_{\mathrm{cr}}+\rho\right)-\frac{1}{2} \int \mathrm{d} \vec{r} \int \mathrm{d} \vec{r}^{\prime} C_{e}\left(\vec{r}-\vec{r}^{\prime}\right) \cdot\left(\rho_{\mathrm{cr}}^{\prime}-\rho\right) \cdot\left(\rho_{\mathrm{cr}}-\rho\right)=0
$$

At $\delta=0$, we get $\rho_{f}=0.93$, with $\eta \simeq 15 \%$. As $\delta$ is increased, $\rho_{f}$ increases (see line FL in fig. $1(\mathrm{~b}))$ and $\eta$ decreases, reaching a value of about $6 \%$ near the point $\mathrm{C}$.

To summarize, we have used a combination of the replica method, liquid theory and density functional theory to obtain the phase diagram of a simple classical fluid in a random pinning potential. Our calculations quantify the effects of the disorder on the crystallization transition of the pure fluid and its glass transition in the metastable "supercompressed" regime. We find that the first-order crystallization transition of the pure fluid changes to a continuous glass transition as the strength of the disorder is increased above a critical value. The phase diagram we have obtained looks qualitatively similar to that of layered type-II superconductors if, instead of increasing the density $\rho$, one decreases the temperature, and if one replaces the disorder strength $\delta$ by the magnetic field $H$. Since our calculations are mean-field in nature, they do not provide a conclusive answer to the question of whether a thermodynamic glass phase exists in $3 d$ randomly pinned classical systems. Further investigations of this issue and extensions of our calculation to layered superconductors and other physical systems would be very interesting. 
We thank M. Mézard, G. Parisi, J. P. Hansen and G. Menon for stimulating discussions. This work was supported in part by the International Program PICS No. 482 between CNRS and Jawaharlal Nehru Centre for Advanced Scientific Research (JNC). Two of the authors (FT and DF) gratefully acknowledge support and hospitality from the Indian Institute of Science and JNC.

\section{REFERENCES}

[1] Seshadri R. and Westervelt R. A., Phys. Rev. B, 46 (1992) 5150.

[2] Andrei E. Y. et al., Phys. Rev. Lett., 60 (1988) 2765.

[3] Blatter G. et al., Rev. Mod. Phys., 66 (1994) 1125.

[4] Zeldov E. et al., Nature, 375 (1995) 373.

[5] Nattermann T., Phys. Rev. Lett., 64 (1990) 2454.

[6] Giamarchi T. and Le Doussal P., Phys. Rev. B, 52 (1995) 1242.

[7] Cubitt R. et al., Nature, 365 (1993) 407.

[8] Menon G. and Dasgupta C., Phys. Rev. Lett., 73 (1994) 1023.

[9] Khaykovitch B. et al., Phys. Rev. B, 56 (1997) R517.

[10] Safar H. et al., Phys. Rev. Lett., 70 (1993) 3800.

[11] Fisher D. S., Fisher M. P. A. and Huse D. A., Phys. Rev. B, 43 (1990) 130.

[12] The question of whether a true thermodynamic glass phase exists in superconductors with random point pinning is controversial - see, e.g., Bokil H. S. and Young A. P., Phys. Rev. Lett., 74 (1995) 3021.

[13] Pitard E. et al., Phys. Rev. Lett., 74 (1995) 4361.

[14] Mézard M. and Parisi G., J. Phys. A, 29 (1996) 6515.

[15] Ertaş D. and Nelson D. R., Physica C, 271 (1996) 79.

[16] Woodcock, L. V. and Angell C. A., Phys. Rev. Lett., 47 (1981) 1129.

[17] Rintoul M. and Torquato S., Phys. Rev. Lett., 77 (1996) 4198.

[18] Ramakrishnan T. V. and Yussouff M., Phys. Rev. B, 19 (1979) 2775.

[19] MÉzard M., Parisi G. and Virasoro M. A., Spin Glass Theory and Beyond (World Scientific) 1987.

[20] Monasson R., Phys. Rev. Lett., 75 (1995) 1170.

[21] Kirkpatrick T. R. and Thirumalai D., Phys. Rev. B, 36 (1987) 5388.

[22] Dasgupta C., Europhys. Lett., 20 (1992) 131.

[23] Sastry S., De Benedetti P. G. and Stillinger F. H., Nature, 393 (1998) 554.

[24] Cardenas M., Franz S. and Parisi G., J. Phys. A, 31 (1998) L163.

[25] The value given in [14] is $\rho_{\mathrm{g}}=1.19$. The discrepancy comes from a different discretization procedure. Given a solution on a real-space grid with spacing $\Delta r$, and a hard-sphere diameter $d$, there is an uncertainty $\Delta \eta / \eta=3 \Delta r / d$ in the value of the associated packing fraction, which accounts for the difference between the two numerical results.

[26] Zerah G., J. Comput. Phys., 61 (1985) 280. 